\title{
El trabajo en red y la cooperación como elementos para la mejora escolar
}

\section{Networking and Cooperation as School Improvement Elements}

\author{
Cristóbal Suárez-Guerrero $^{1^{*}}$ \& José Luís Muñoz Moreno \\ ${ }^{1}$ Universitat de València, Valencia, España. \\ ${ }^{2}$ Universitat Autònoma de Barcelona, Barcelona, España.
}

Recibido 31-12-16

Aprobado 30-03-17

Correspondencia

Email: cristobal.suarez@uv.es

\section{Citar como:}

Suarez-Guerrero, C., \& Muñoz, J. (2017). El trabajo en red y la cooperación como elementos para la mejora escolar. Propósitos y Representaciones, 5(1), 349 - 402 doi: http:// dx.doi.org/10.20511/pyr2017.v5n1.150

(C) Universidad San Ignacio de Loyola, Vicerrectorado de Investigación y Desarrollo, 2017.

(๕) BY-NC-ND Este artículo se distribuye bajo licencia CC BY-NC-ND 4.0 Internacional (http://creativecommons.org/licenses/by-nc-nd/4.0/). 


\section{Resumen}

La escuela es un entorno enriquecido de aprendizaje, pero no es el único entorno educativo. La misión educativa de la escuela debe contemplar la coordinación escuela-familia como rasgo de su proyecto social. Buena parte de este puente se fundamenta en el diálogo desde la participación de la familia con el desarrollo educativo integral, base de la noción democratizadora de la escuela. Por ello, esta aportación aborda el trabajo en red como elemento para la mejora escolar desde la cooperación. Se focaliza en el trabajo en red al servicio del aprendizaje y el conocimiento y en los equipos cooperativos como unidad de trabajo en red. Una muestra de esta forma de trabajo se concreta en dos experiencias relevantes en el contexto español: el "Plan Integral del Polígono Sur” (Sevilla) y "Deporte y Escuela” (Ripollet). Las principales conclusiones ponen de manifiesto que la participación abierta, la búsqueda del consenso y la gestión de la interacción son instrumentos útiles para fomentar la cooperación y el trabajo en red en los diversos contextos de intervención y los distintos escenarios de actuación.

Palabras clave: Escuela, familia, cooperación, trabajo en red, participación.

\section{Summary}

The school is an enriched learning environment, but it is not the only educational environment. The educational mission of the school should take into account the school-family coordination as a feature of its social project. A great part of this bridge between school and family is based on dialogue through the participation of the family in the comprehensive educational development, which is the basis of the democratizing notion of school. Therefore, this work addresses networking as a school improvement element through cooperation. It focuses on networking for learning and knowledge, and on cooperative teams as a networking unit. An example of this way of work can be specified in two relevant experiences in the Spanish context: the "Poligono Sur Comprehensive Plan" (Sevilla) and "Sport and School" (Ripollet). The main conclusions shows that open participation, consensus- 
building and interaction management are useful tools to foster cooperation and networking in the various contexts of intervention and the different action scenarios.

Keywords: School, family, cooperation, networking, participation. 


\section{Introducción}

No se puede entender la escuela como una abstracción social. La escuela está influenciada por su entorno social, al que se debe y que define sus necesidades, problemas y también proporciona algunas soluciones. La teoría sociocultural es clara a este respecto (Vigostky, 2000): el entorno, tanto en el aprendizaje como en la construcción cultural, no es una entidad neutra, sino que forma parte de la explicación y el desarrollo de los procesos que allí se alojan. La misión de la escuela, centrada solamente en la función de enseñanza, se debilita si no se concibe la experiencia de aprendizaje como un evento amplio, que atañe tanto a la sociedad como al profesorado y a las familias. Decir que en la escuela se educa puede sonar desproporcionado si únicamente se asiste para aprender contenidos y se vive de espaldas a la realidad y la coordinación con las familias. La escuela tiene que pensar en opciones más abiertas al diálogo educativo permanente con su entorno.

La escuela y las familias pueden mantener un diálogo fructífero desde la participación educativa en el desarrollo y la mejora de la educación (Vigo \& Julve, 2016; Llevot \& Bernad, 2015; Vicent, 2014). Este es uno de los retos principales en la democratización de la sociedad, porque ya no es suficiente con destinar recursos para mejorar la calidad educativa, sino que también se precisa generar oportunidades de participación en el ámbito de la educación y desde la suma de los intereses colectivos. Una educación democrática, de acuerdo con Bolívar (2005), debe ser constitutiva, como fin y medio, de la educación pública.

La interacción social, el debate público, el respeto y la atención a la diversidad, son fundamentales en la educación. El ejercicio del derecho a la educación tiene que procurar una efectiva participación de las familias, también en los procesos de toma de decisiones (Morin, 2001). Para Gairín y San Fabián (2005) este es un indicador de normalización democrática y para Fernández (1997) constituye una base para la democracia y el desarrollo. Los estudios sobre escuelas eficaces ya demostraron que la implicación de las familias en la educación influía favorablemente en el rendimiento académico 
del alumnado. Algunas razones que apoyan la tesis de la colaboración entre escuela y familias coinciden con las señaladas por Mayordomo (1999): necesidad de extensión, definición y profundización en los principios de democratización; sentido de responsabilidad y colaboración; apoyo a la creatividad y al espíritu crítico como fuente de vitalidad; control de un uso adecuado de los recursos financiados con fondos públicos; y contribución al aprendizaje democrático y a la adquisición de un sentido de comunidad.

Un contexto adecuado para esta colaboración debiera procurar la formación necesaria para "saber participar", la organización de medios para "poder participar" y la motivación para "querer participar" (Martín \& Gairín, 2006). Siempre a partir de respetar al otro y el reconocimiento mutuo, la orientación a procesos y resultados, la inclusión (Lozano, Castillo, Cerezo, Angosto \& Alacaraz, 2014), la voluntariedad, la igualdad de oportunidades y la equidad. Los beneficios de ello quedan asociados a una mayor comprensión de ciertas cuestiones y situaciones de lo qué se hace, un acercamiento a la responsabilidad por los problemas y las necesidades colectivas, una reducción de las injusticias y las diferencias por el reparto de poder, una mejor transparencia y control social, un incremento de la confianza y más legitimidad ante los procesos desarrollados (Muñoz, 2012).

La escuela educa y debe garantizar que el trabajo que desempeña en materia de valores y competencias asegure una óptima cohesión y convivencia en la vida escolar y cuando el alumnado sea adulto y tenga responsabilidades directas en los ámbitos social, laboral, familiar, político, etc., porque el desarrollo de la ciudadanía depende, en gran medida, del nivel de cohesión y convivencia existente. La consecución de este propósito atañe a la escuela, pero también a las familias y a la sociedad desde la lógica de la corresponsabilidad con la educación (Soler, 2006). Según Apple y Beane (2005) conviene ir más allá de la escuela, preocupándose por las injusticias y las desigualdades sociales y entendiéndola como un lugar de transformación social y no como una entidad sumida en sí misma. Por tanto, ¿desde qué visión se puede articular la coordinación y la corresponsabilidad entre 
escuela y familias para lograr un impacto educativo más significativo en el desarrollo integral del alumnado en edad escolar? Este es el problema objeto de estudio sobre el que reparamos aquí.

La escuela como creación social, promovida y articulada para el cumplimiento de los fines establecidos por la sociedad, ha de ser sensible a las necesidades y las demandas socioeducativas existentes. La participación de las familias en ella sirve para hacer conscientes los intereses legítimos que todos pueden tener y evitar la transmisión de prejuicios, estereotipos o actitudes discriminatorias y excluyentes. Por ello, y comprendiendo que la escuela juega un papel decisivo en la formación de la ciudadanía y la inclusión (Bolívar, 2005), cabe pensar que el aula es también síntesis cultural y espacio de creación de pensamiento (Gairín, 1998). Hay que reconocer la necesidad de concebir la escuela como promotora de un proyecto cultural comunitario que recoge las propuestas de coordinación entre escuela y sociedad (Muñoz \& Tolosana, 2010). En esta contribución se caracteriza el trabajo en red como elemento para la mejora de la función de la escuela desde la cooperación con la sociedad y, al respecto, se muestra su viabilidad a través de dos experiencias relevante en el contexto español: el "Plan Integral del Polígono Sur" (Sevilla) y "Deporte y Escuela” (Ripollet).

\section{Trabajo en red al servicio del aprendizaje y el conocimiento.}

Avanzar hacia una escuela que aprende y promueve la creación y gestión de conocimiento (Revilla \& Pérez, 1998), desde la participación de la comunidad educativa, requiere de la incorporación de metodologías y dinámicas de trabajo en red que posibiliten la colaboración en la consecución de los fines educativos (Bassedas, 2007). De esta forma, el trabajo en red supone colaborar de forma sistemática, coordinada y complementaria, tejiendo relaciones y complicidades en espacios comunes, abiertos y diversificados, para lograr esos fines a partir de actuaciones concretas. Se inspira en el aprendizaje mutuo y colectivo, mediante la reflexión crítica y la 
autoevaluación, pero también en el crear y gestionar conocimiento operando conjuntamente (Rodríguez-Gómez, 2015).

Actualmente las posibilidades de la escuela por impulsar la colaboración sirven a funciones tan diversas como, por ejemplo, mejorar el conocimiento recíproco, denunciar situaciones injustas, reivindicar la igualdad de oportunidades y la equidad, compartir recursos, participar en proyectos comunes, establecer redes u otros compromisos (Muñoz, Rodríguez \& Barrera, 2013). Desde ahí, es posible articular redes interpersonales entre iguales, redes colectivas entre estamentos y asociaciones, redes internas o externas a la escuela, redes de servicios y otras.

El trabajo en red orientado a la creación y gestión de conocimiento necesariamente debe pasar por distintas fases, tal y como señalamos aquí: a) la planificación del diseño de actuaciones que se implementarán en base a las metas propuestas; b) la distribución de tareas, concretando las funciones y las actividades que se pondrán en juego, democrática y participativamente; c) el desarrollo, marcando las interrelaciones entre los componentes de la red y facilitando la horizontalidad en la distribución de poder; d) la coordinación, para una mayor operatividad, con canales abiertos de comunicación y toma de decisiones que generen un clima positivo de trabajo, respeto a la autonomía propia y una mejor participación de todos; e) la evaluación útil y alineada con la finalidad a la que se sirve, sistemática, valorativa, decisoria, prospectiva, fundamentada en la recogida de información rigurosa y encaminada a tomar decisiones, emitir juicios de valor y sugerencias respecto al futuro.

En coherencia, conviene delimitar proyectos comunes que permitan crecer, desarrollarse conjuntamente y disponer de espacios para hacerlo (Gairín, 2004). Sin embargo, hay que tomar conciencia de que trabajar en red también consume tiempo, a veces sobrecarga de tareas e incluso puede provocar confusión ante determinados tipos de liderazgo poco pertinentes (Ubieto, 2009). 
Un funcionamiento adecuado del trabajo en red procura a quiénes se implican y participan, suficiente información y transparencia, consulta previa, negociación de posicionamientos y considera y analiza todas las propuestas, evitando la obstrucción desde bandos, evaluando, delimitando acuerdos y acciones prácticas y priorizando las respuestas a las necesidades y los intereses colectivos. Estos elementos conectan con otros factores de éxito tales como asumir una cultura de intervención en red, responsabilizar y comprometer a los actores, poner en marcha sistemas de comunicación, dar correspondencia a los planes de trabajo, participar en los procesos de toma de decisiones o tender puentes de entendimiento. Por consiguiente, la confianza, el diálogo y el consenso aquí resultan más que imprescindibles $\mathrm{y}$, en este sentido, cualquier proceso que se inicia debe sostenerse sobre las bases de la libertad de acción, la igualdad de trato, la lealtad y el compromiso en los términos de la colaboración, la participación en políticas conjuntas, el respeto a la diferencia, la inclusión y la complementariedad (Muñoz, 2014).

Colaboración y participación dotan de sentido y significado el trabajo en red, especialmente si se conciben como una actuación conjunta y comprometida, aplicable a situaciones distintas (profesionales e institucionales) y desde diferentes niveles de contenido en beneficio del aprendizaje y el conocimiento. Son claves cuando los diversos estamentos de la escuela tienen que mantener sinergias ante el desempeño de ciertas propuestas y políticas educativas. A su vez, aprendizaje y conocimiento acontecen procesos de colaboración y participación dónde compartir propósitos desde la implicación flexible y solidaria de todos en su consecución, así como en las tareas que se desprenden (Koper, 2009).

Esto refuerza una cultura común a favor del aprendizaje individual y organizacionaly de la creación y gestión de conocimiento colectivo. Esasícomo la escuela contribuye a modificar las formas de funcionar institucionalmente que lo precisan, comparte valores con los demás y preocupaciones comunes, se abre al cambio y la innovación o genera compromisos que derivan en retos ante la sociedad del conocimiento en la que se inscribe (Hargreaves, 
2003); sólo posibles desde planteamientos cooperativos capaces de remar en la dirección de la mejora educativa permanente.

La escuela, desde esta óptica, puede cobrar un mayor protagonismo institucional en su entorno social y con la comunidad dónde se inserta. Progresa y se desarrolla a través del conocimiento, creándolo, socializándolo, evaluándolo, renovándolo y actualizándolo (Blázquez, 2013). El conocimiento adquiere relevancia en tanto que es colectivo y se comparte para resolver las problemáticas planteadas a la escuela y en la medida que proporciona la oportunidad a la comunidad educativa de aprender. En suma, se trata de que el conocimiento pase de vincularse a la competitividad para ganar en compartición y transversalidad.

En cualquier caso, la construcción de una escuela generadora de aprendizaje y conocimiento tiene que partir de una visión a medio y largo plazo y de la convicción de progresar gradualmente. La responsabilidad ejercida por la escuela y su respectiva comunidad educativa ante la creación y gestión de conocimiento, puede situarla en el trayecto de la mejora y el desarrollo, en lo más próximo, en la capacidad de cooperar y trabajar en red para promover y facilitar el aprendizaje constante de todos y la innovación (Nagles, 2007). De las personas, de la comunidad y de la organización. Más aún cuando la inversión en aprendizaje y conocimiento reduce determinadas barreras socioeducativas desde un punto de vista social, refuerza los planteamientos democráticos desde una perspectiva política y genera una mejor productividad desde una visión económica.

El trabajo en red focalizado en estos aspectos va a cobrar su máximo sentido en tanto y cuanto sepa ponerse al servicio de los procesos de transformación de la escuela y supere el mero y simple conocimiento superficial de las cosas. La relación entre el desarrollo de la escuela y la mejora de la comunidad educativa no puede quedar al margen de las transformaciones organizativas que la escuela pueda impulsar si realmente aspira a modificaciones profundas de los procesos formativos y de enseñanza y aprendizaje (Senge, 1990). Sin lugar a dudas, crear y gestionar conocimiento refuerza las metas educativas 
establecidas colectivamente y la articulación de procesos cooperativos de revisión y mejora permanentes.

\section{Los equipos cooperativos como unidad de trabajo en red.}

La redes son nodos de coordinación que operan en torno a metas comunes y se comunican entre sí para tal efecto (Suárez y Gros, 2013). La cooperación como estrategia social de desarrollo (Santos Guerra, 2009) no ha terminado de calar en las formas de comprensión pedagógica. A pesar de la ya larga tradición en el aprendizaje (Pujolàs, 2004; Johnson, Johnson \& Holubec, 1999; Slavin, 1999) como modelo social de enseñanza (Joyce \& Weil, 2002), la cooperación sigue siendo una opción alternativa en la forma de organizar el aula y en la manera de trabajar en la escuela. Aún su valor didáctico e impacto en el aprendizaje (Suárez, 2007), la cooperación desempeña un rol importante como estrategia de coordinación y organización social de la escuela (Fernández Enguita \& Terrén, 2008). El reto de la cooperación y el trabajo en red, por lo tanto, es también un reto organizacional (Rudduck \& Flutter, 2007) que la escuela debe asumir como rasgo de calidad.

Ese reto puede cifrarse de acuerdo a este dilema: la escuela como institución para la que enseñar $-y$ educar- es un fin en sí mismo, no un medio para nada, puede optar por aislarse de ello y oponerse o bien por tratar de fomentar la cooperación con educadores e introducir un cierto sentido compartido en la maraña de oportunidades y reclamos que hoy se presentan ante la infancia y la juventud. Lo primero supone la burocratización y el aislamiento de la organización; lo segundo, el trabajo en red (Fernández Enguita, 2012). La relación profesorado-alumnado en el aprendizaje y las relaciones verticales en la escuela son formas acostumbradas en la práctica educativa que, en definitiva, no son las únicas. Si estas formas subsisten es porque, entre otros factores, se mantiene vigente la forma de representar la educación o, concretamente, la relación educativa.

Toda opción de enseñanza -y educativa- entraña una opción de relación, la pregunta que se puede formular ahora es ¿toda alternativa de relación 
educativa es igualmente significativa y beneficiosa para la experiencia formativa? o ¿es posible pensar en algún tipo de relación educativa cualitativamente más ventajosa? El cambio supone la práctica, pero también la reelaboración conceptual sobre la forma de pensar la relación entre los agentes educativos, punto de quiebra para detectar otros umbrales de desarrollo.

Un error educativo suele ser un error de concepción relacional (García y García, 2001). El desatino relacional suele tener consecuencias en distintos niveles del proceso educativo. Por eso, cuando se plantea una opción educativa estamos, de forma explícita o implícita, frente a una opción relacional sobre qué y cómo se pueden enriquecer los entornos de aprendizaje gracias a la educación. Aquí es donde adquiere sentido la cooperación como estrategia de desarrollo educativo (Mir, 1998). Pero no todo queda en la enunciación, sino que hay que preguntarse: ¿qué es aquello que cambia en una situación educativa cuando se piensa como coordinación cooperativa?

En primera instancia, la actividad cooperativa es una acción distinta a los procedimientos competitivos e individualistas que se destacan, normalmente, por valorar el aislamiento y la incomunicación como factores de desarrollo educativo. Educar bajo la clave cooperativa significa educar bajo la consigna de una nueva unidad: el equipo (Suárez, 2010).

Y un equipo no es un grupo. Hay muchas agrupaciones, pero pocas funcionan como equipos. Tampoco interactuar es un rasgo diferenciador nítido de un equipo, muchos individuos pueden interactuar, debatir o discrepar y seguiría siendo interacción. La opción relacional que está detrás de un equipo es la interacción cooperativa que tiene sus distinguidos rasgos y formas de desarrollo. Un equipo es la unidad básica de la intersubjetividad entre iguales (Suárez, 2010), un concepto desde donde se puede representar, comprender y analizar la interacción cooperativa en el aula y en la escuela. Para que las personas pasen de formar grupos a formar equipos es necesario, coincidiendo con Pujolàs (2004, p. 77), reparar en los siguientes rasgos: 
- Si están unidas de verdad y si tienen alguna cosa que las une fuertemente (la pertenencia al mismo equipo, el objetivo que persiguen, etc.).

- Si hay una relación de igualdad entre ellos, si nadie se siente superior a los demás, si todos son valorados y si se sienten valorados por sus compañeros.

- Si hay interdependencia entre ellos y si lo que afecta a un miembro del equipo importa a todos los demás.

- Si no hay una relación de competencia entre ellos, sino de cooperación, de ayuda y de exigencia mutua y si ayudar a un compañero repercute favorablemente en uno mismo y en todo el equipo.

- Si hay una relación de amistad entre ellos y un vínculo afectivo que los lleva a celebrar juntos los éxitos conseguidos entre todos los miembros del equipo.

Para ser más concretos y aportar una visión que dé paso a la práctica se pueden plantear los rasgos característicos de un equipo cooperativo. Tomando como base los principios de la acción cooperativa (Suárez, 2011; Johnson, Johnson \& Holubec, 1999), es posible identificar hasta cinco dimensiones básicas configuradoras de un equipo cooperativo. En consecuencia, un equipo cooperativo actúa como unidad básica de intersubjetividad, siempre y cuando se articulen las siguientes cinco dimensiones.

a. Interdependencia positiva. Los individuos identifican que el logro de su objetivo depende del esfuerzo de todos los miembros del equipo para alcanzar la meta compartida, puesto que mejorar el trabajo de todos supone también mejorar el suyo propio. Esto sucede al enfocar, como propósito inequívoco de la relación educativa, la conciencia de que el éxito personal depende del éxito del equipo. Si uno fracasa, entonces fracasan todos los demás.

b. Responsabilidad individual y de equipo. Cada miembro del equipo asume su responsabilidad, pero a su vez hace responsables a los demás del trabajo que deben cumplir para alcanzar los objetivos comunes a todos. 
En este caso, el sentido de responsabilidad con la tarea personal, así como con la tarea en conjunto, es el factor que contribuye a no descuidar la parte y el todo.

c. Interacción estimuladora. Los miembros del equipo promueven y apoyan el rendimiento óptimo de todos los integrantes a través de un conjunto de actitudes que incentivan tanto la motivación personal como la del conjunto. Las ayudas, los incentivos, los reconocimientos, los alientos y la distribución de los recursos contribuyen a crear este clima de confraternidad en torno al objetivo común.

d. Gestión interna del equipo. Los miembros del equipo planifican y coordinan sus actividades de manera organizada y concertada a través de planes y rutinas, como también mediante la repartición de funciones para alcanzar la meta común de equipo. Esto implica que cada miembro despliegue acciones para estimular un funcionamiento efectivo del equipo, como por ejemplo la toma de decisiones, la gestión del tiempo, la superación de problemas, el liderazgo o la regulación de turnos de trabajo.

e. Evaluación interna del equipo. El equipo valora constantemente su funcionamiento interno en base al logro de la meta conjunta, así como el nivel de efectividad de la participación personal en la dinámica cooperativa. Esto requiere analizar y valorar en qué medida se estarían logrando concretar las metas de aprendizaje compartidas, pero también cómo se han desempeñado cooperativamente todos y cada uno de los miembros.

Cada equipo goza de distintos sentidos e intensidades, aunque las cinco dimensiones pueden contribuir a diseñar, desarrollar y evaluar actividades de trabajo entre escuela y familias. Se trata de representar un equipo como la oportunidad de cooperación. Por ello, para mejorar la escuela desde la cooperación no basta con estar juntos, o con compartir el mismo espacio educativo, sino que hace falta pensarla como unidad de intersubjetividad poniendo en práctica estas dimensiones cooperativas.

\section{Concretando la cooperación en la práctica.}

La cooperación entre escuela y familias habitualmente se ha formalizado a través de la representación en las estructuras de participación, o mediante la implicación en asociaciones e incluso con un carácter informal. Todas 
las posibilidades son reales, no excluyentes, pero sería oportuno que compartieran realidades, políticas y planteamientos (Gairín, 2004a).

La escuela, situada en un escenario social y político verdaderamente democrático, precisa disponer de formas de cooperación con su entorno, de control participativo, de evaluación colaborativa y de gobierno conjunto y en común (McDonald, 1995; Martínez, 1998; Martín, 2000; Páez, 2015). De acuerdo con Epstein (1990), la cooperación entre escuela y familias podría concretarse en la práctica alrededor de las siguientes áreas de trabajo: la escuela como fuente de apoyo a las familias para que éstas puedan cumplir con sus obligaciones educativas básicas; las familias como fuente de soporte a la escuela para que ésta alcance una intervención educativa mejor y más eficaz; la cooperación de las familias en la escuela para desarrollar actividades complementarias de soporte y apoyo; la participación de las familias en actividades de aprendizaje con sus hijos e hijas y en la organización desde los órganos de gestión; y la relación de las familias de la escuela con otras comunidades educativas e instituciones sociales.

De hecho, en el caso de la cooperación de las familias para la organización y gestión de la escuela, se requiere asumir la diversidad de estructuras y la variedad de contextos existentes. Además, se necesita que la escuela analice los cambios en las funciones socializadoras y educativas que se presentan en las distintas estructuras familiares (nuclear, reconstituida, extendida, monoparental, homoparental, ensamblada y de hecho) para adecuar a ellas sus planes, programas y proyectos, así como para clarificar las funciones que corresponden a cada colectivo y hacer efectiva una acción coordinada (Ordóñez, 2015).

Es fundamental la cooperación entre los sectores sociales que inciden en los procesos educativos, comenzando por la corresponsabilidad activa de las familias por ser la primera y más genuina instancia educadora, tal y como se ponía de manifiesto en las conclusiones del reciente "XXIII Encuentro de Consejos Escolares Autonómicos y del Estado", celebrado en Santander (España) y que se focalizó en la relaciones entre familia y escuela (MECD, 
2015). Potenciar esta cooperación es un ejercicio de responsabilidad socioeducativa pero también un requisito para los modelos de gestión educativa. En relación a ello, podemos destacar dos experiencias relevantes en el contexto español, por su novedad y originalidad.

La primera es el proyecto de intervención socioeducativa y familiar concebido dentro del "Plan Integral del Polígono Sur" (Sevilla), aprobado por el Consejo Gobierno de la Junta de Andalucía el 20/12/2005․ La población de esta zona de Sevilla asciende a aproximadamente 50.000 personas y se concentra en unas 7.000 viviendas en una superficie de 145 hectáreas. La población, que fue llegando a esta zona desde la década de los años 60 del siglo pasado, posee una gran diversidad de procedencias, culturas, lenguas, formaciones y ocupaciones de las familias. Es en esta realidad que nace el movimiento vecinal del Polígono Sur, asentando todas sus iniciativas en tres pilares: integralidad, para encarar de forma conjunta y no sectorial los problemas; adaptación territorial, para entender los problemas y las soluciones como propios al entorno; y participación, para tener en cuenta e involucrar todas las visones posibles de la realidad.

El “Plan Integral del Polígono Sur” implica un modelo de participación, gobierno y ciudadanía que tiene dos objetivos claros. Por un lado, desarrollar un nuevo modelo de gestión de las políticas públicas, específico para el Polígono Sur, basado en altos niveles de coordinación de la acción pública y de participación activa ciudadana y, por otro, mejorar las condiciones de vida en el Polígono Sur, basada en cuatro ámbitos básicos de actuación integrada: urbanismo y convivencia vecinal, inserción sociolaboral y promoción de la iniciativa económica, intervención socioeducativa y familiar y salud comunitaria. Es aquí donde se inscribe la idea educativa de cooperación entre escuela y familias, en un lugar donde la respuesta educativa forma parte de una visión más amplia y global de sociedad. 
La idea educativa (Comisionado para el Polígono Sur, 2010) es dar una respuesta educativa integral y comunitaria. Se concreta en cuatro programas de acción para disminuir el fracaso escolar, mejorar la organización escolar, optimizar la infraestructura educativa y potenciar la educación permanente. En todos los programas hay acciones específicas de coordinación entre escuela y familias que se plasman en un singular modelo de gestión (Figura $1)$.

\begin{tabular}{|c|c|c|}
\hline \multicolumn{3}{|c|}{ MODELO DE GESTIÓN } \\
\hline $\begin{array}{l}\text { COMISIÓN MDXTA DE } \\
\text { OESARROLL, SEEUIMIENTO Y } \\
\text { EYALUACION }\end{array}$ & GRUPO TECXICO & EQUIPO DE TRABANO DE ZONA \\
\hline $\begin{aligned} & \text { Presidencia: comisionado } \\
&> \text { Director/a General Innovación } \\
&> \text { Delegado/a provinclal } \\
&> \text { Delegadova municipal } \\
&> \text { Secretaria } \\
&> \text { Vocales: } \\
& \text { - Jete/a servicio OEAD } \\
& \text { (consejeria) } \\
& \text { - Jeta/e servicio or. Ed } \\
& \text { (delegación) } \\
& \text { - Dirección técnica comisionado }\end{aligned}$ & $\begin{array}{l}\text { Presidencia: jefa/e servicio or. Ed } \\
\text { (delegación) } \\
>\text { Secretaria } \\
>\text { Jefe/a servicio OEAD (consejeria) } \\
>\text { Dirección técnica comisionado } \\
>\text { Representante de la Delegación } \\
\text { Municipal de Educación } \\
>\text { Jefatura de inspeccion } \\
>\text { Coordinadoria equipo educativo } \\
\text { comisionado } \\
>\text { Comisión ampliada } \\
\text { - Jefaturas de servicio } \\
\text { - Direcciones de centros } \\
\text { educativos }\end{array}$ & $\begin{array}{l}>\text { Presidencia: inspección zona } \\
>\text { Secretaria } \\
>\text { Responsable de compensatoria } \\
\text { (delegación) } \\
>\text { Area socioeducativa del } \\
\text { comisionado } \\
>\text { Direccion de centros educativos } \\
>\text { Coordinador/a del EOE } \\
>\text { Asesor de referencia del CEP }\end{array}$ \\
\hline & IÓN SOCIOEDUCATIVA Y FAR & IAR \\
\hline
\end{tabular}

Figura 1. Modelo de gestión del "Plan Educativo del Polígono Sur" (Comisionado para el Polígono Sur, 2010, p. 55).

Entre los mecanismos más formales de gestión educativa de la relación escuela y familias de la experiencia del Polígono Sur se puede destacar la "Comisión Socioeducativa y Familiar". Este estamento se concibe como un órgano territorial de gestión y participación educativa para "establecer un espacio de trabajo en el que están presentes los servicios públicos, las AMPAS y entidades de actuación socioeducativa, con el que se pretende aumentar 
la coordinación entre las administraciones para mejorar sus actuaciones y lograr la participación activa de los vecinos y vecinas" (Comisionado para el Polígono Sur, 2010, p. 53). Con esto se quiere garantizar la participación de las familias no únicamente en el acompañamiento educativo, sino también en las decisiones de la comunidad educativa. Para ello se trabaja por subcomisiones donde las familias tienen presencia en ámbitos como la escolarización, la convivencia, la formación, la educación en los espacios públicos y las infraestructuras.

La segunda experiencia es "Deporte y Escuela" (Ajuntament de Ripollet, 2016) que, creada y desarrollada por el Patronato Municipal de Deportes del municipio de Ripollet ${ }^{2}$ (provincia de Barcelona), presenta un modelo de deporte escolar que trabaja en la parte más lúdica de la práctica deportiva, fomentando valores socioeducativos, poniendo el acento en la diversión y no en la competición y contando con la participación activa de las escuelas y las familias, pero también de otros departamentos de la Administración local (Figura 2).

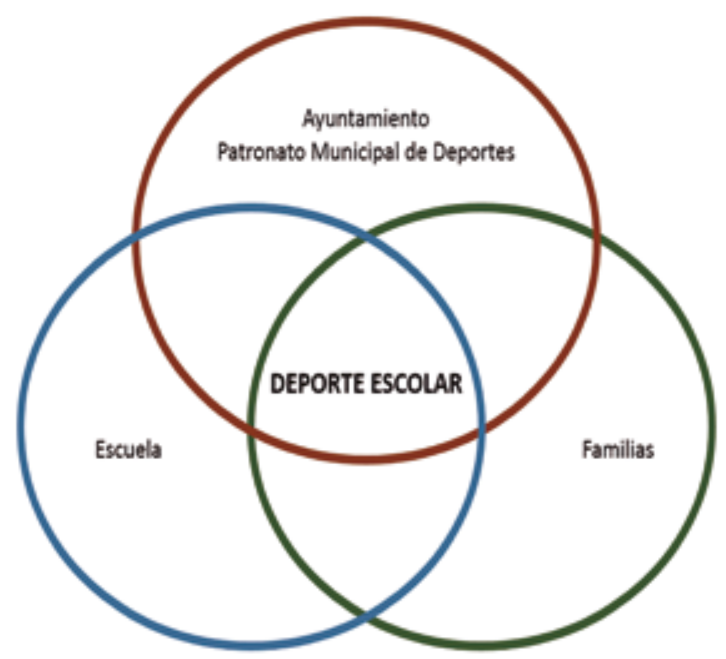

Figura 2. Agentes para la cooperación en el deporte escolar (Ajuntament de Ripollet, 2016). 
El objetivo común que reúne a todos los agentes es que todos los niños $\mathrm{y}$ todas las niñas del municipio puedan realizar deporte fuera de las horas de escuela, de una manera regular y con calidad, aumentando su conocimiento deportivo y experimentando distintas modalidades deportivas. De forma relevante, la experiencia incorpora cambios en los sistemas de competición en los deportes de equipo: se añade un protocolo de saludo al rival; las cuatro partes de los partidos cuentan con un punto en el resultado final, evitando derrotas superiores al 4 a 0 ; se integra un quinto tiempo amistoso que se juega con equipos mezclados y dónde los rivales pasan a ser compañeros; y en las actas no se contabilizan las acciones individuales. En el caso de los deportes individuales: se eliminan las clasificaciones; se suprimen los sistemas de juego por eliminación para que los jóvenes deportistas jueguen el mismo tiempo; y compiten juntos niños y niñas.

El éxito de la práctica, que implica a alrededor de unos 1.200 escolares, necesita pues de la cooperación entre escuelas, familias y Ayuntamiento en aspectos inspiradores tales como (a partir de Gairín, Castro, Díaz \& Muñoz, 2012):

- El fomento de la deportividad, el deportista participa de la competición por su satisfacción, reconocer y valorar las actuaciones de los participantes, no obligar al deportista a realizar un deporte que no desea, enseñar al deportista a admitir los resultados sin que se desilusione o condenar el uso de la violencia en todas sus formas.

- No desprestigiar al deportista que comete un error o pierde, evitar fomentar la competición al límite de la deportividad, animar al deportista y no generarle una presión excesiva, no centrarse en si gana o pierde y sí en el esfuerzo y la implicación o enseñar al deportista la diferencia entre deporte escolar y deporte profesional.

- Respetar al rival, no intimidarlo, no ridiculizarlo, responder con indiferencia a provocaciones, no resolver el desacuerdo con 
violencia, no hacer gesticulaciones groseras o no utilizar un lenguaje indebido.

- No protestar al árbitro, respetar sus decisiones, animar al deportista a competir según el reglamento, mostrar agradecimiento al árbitro o no cuestionar su honestidad.

- Respetar las decisiones técnicas y no criticarlas, no protestar ni discutir al cuerpo técnico y entrenadores, mostrar agradecimiento al entrenador o no presionar a los entrenadores.

- Respetar los derechos, la dignidad y los valores de todos los implicados en la competición deportiva, sin distinción de ningún tipo; y respetar las instalaciones deportivas, haciendo un buen uso de ellas.

Se trata así, cooperando entre todos, de asumir responsabilidades con el juego limpio, el buen comportamiento y más allá del simple respeto a las reglas: amistad, respeto al adversario y espíritu deportivo como propiciadores de cohesión y convivencia sociales, pero también de ética.

De hecho, tanto la anterior experiencia como ésta, sólo son posibles desde actitudes abiertas de la escuela, la inclusión con el entorno y un marco de relaciones para trabajar en red dónde estén más que presentes las familias. Lo que se persigue no es otra cosa que compartir propósitos y materializar la voluntad de cooperar en un proyecto común para asimismo compartir conocimiento y operar desde la interdependencia entre los actores, la interacción sostenida y la autoridad soberana (Muñoz, 2012; Muñoz, 2009).

\section{Conclusiones}

La educación como derecho, y desde la cooperación entre escuela y familias, debe comprenderse desde la noción de participación social y de aprendizaje a lo largo de toda la vida. De acuerdo con Esteban (2015) se debe enmarcar dentro de una cultura participativa, que ha de instaurarse progresivamente, realizando pasos pequeños pero seguros y continuos. 
La cooperación entre escuela y familias no puede ser entendida como una alternativa marginal o un ensayo novedoso, es ante todo una práctica educadora en toda regla, un pilar sin el cual no es posible hablar de educabilidad. La escuela no puede permitirse pensar la finalidad educativa, ni con ello la parte más concreta relacionada con el éxito o el fracaso en los aprendizajes, si no da voz a las familias y busca mecanismo de implicación conjunta.

Visto este reto de corresponsabilidad, la gestión educativa debe partir de la cooperación entre escuela y familias en favor de la igualdad de oportunidades, la equidad, la inclusión y, en definitiva, la justicia social. Evolucionar en este trayecto es caminar hacia una escuela que aprende y que estima clave la cooperación y el trabajo en red como posibilitadores de nuevas formas de implicación de la ciudadanía en las decisiones colectivas sobre la complejidad de la educación como asunto público. La concreción de la cooperación en la práctica cotidiana exige, por ello, que tanto escuela como familias tomen en consideración los intereses del conjunto, respetando siempre los propios de los demás, sin vulnerarlos y cumpliendo satisfactoriamente con las responsabilidades que les han sido atribuidas. La intervención, así y en todo momento, queda orientada hacia el logro de mejores condiciones educativas para todos y todas y en los diversos niveles y esferas.

Aunque la cooperación tradicional entre la escuela y las familias se concrete a través de fórmulas abiertas de participación o asociación espontánea, es sustancial que ambas compartan políticas y diseñen proyectos conjuntos. Esto es pasando a formar parte de una visión de la gestión educativa más amplia que la estricta gestión escolar. Muestra de este desarrollo son las experiencias tratadas, "Plan Educativo del Polígono Sur" y "Deporte y Escuela", que permiten realmente hablar de los retos, los problemas y las posibilidades que afrontan los modelos de coordinación educativa entre escuela y familias. En ambos casos se hace patente la coordinación cooperativa y en red entre escuela y familias, pero no solo como sinónimo de un mejor rendimiento académico, sino también como gestión de fenómenos 
significativos -organizados con intencionalidad educativa- que promueven la participación y la coordinación de los agentes educativos en beneficio de unas experiencias educativas más amplias. Iría ello en la senda de lo ya apuntado por Egido (2015) cuando destaca que el éxito de la relación entre escuela y familias y sus implicaciones en la práctica de intervención mucho tienen que ver con la presencia de estos rasgos: partir de la consideración de padres y profesores como iguales, un clima de diálogo y confianza mutua; la escuela adopta un papel proactivo ante la cooperación; desarrollar acciones para conseguir la participación de todas las familias; diseñar fórmulas de cooperación adaptadas a las distintas etapas educativas; destinar tiempo y esfuerzo a motivar y a formar a todos los sectores implicados; y hacer una plantificación estratégica profundizando en formas de cooperación realistas, flexibles y duraderas.

Representan respuestas oportunas a las necesidades educativas que cada entorno describe. Por ello, se pueden derivar de ellas criterios y tendencias recomendables, considerando que no son fórmulas cerradas en sí mismas. Ambas experiencias no han sido producto del azar o de la contingencia administrativa, sino que forman parte de planes articulados desde una visión holística de la educación donde escuela y familias son imprescindibles.

La cooperación y la participación dotan de sentido y significado el trabajo en red. De forma concreta, la cooperación entre escuela y familias puede acaecer como equipos de trabajo cooperativo y en red que, entre otros aspectos de la organización educativa, no descuide la interdependencia positiva cimentada en la meta compartida, la responsabilidad individual y de equipo que permite no olvidar la parte y el todo, la interacción estimuladora entre los miembros pensada como diálogo y motivación interpersonal, la gestión interna del equipo basada en acciones estimuladoras de un funcionamiento efectivo y la práctica de una evaluación interna del equipo que valora los productos y los procesos de trabajo. Pensar la educación abriendo estos puentes de cooperación con las familias permitiría hablar de la calidad de la educación, no solamente como un indicador de rendimiento 
académico, que quizás sea sólo la punta del iceberg, sino como entrada a las raíces educativas que la escuela a veces ha dejado de lado al pensar en el éxito educativo.

La coordinación entre escuela y familias no es, por lo tanto, una práctica coyuntural ni incidental que pueda relegarse a un segundo plano. Forma parte de una visión efectiva del trabajo educativo actual (Vigo, Dieste \& Julve, 2016) y define una línea de investigación educativa cada vez más importante cuando se asume la idea de que la escuela no es el único entorno educativo (Páez, 2015; Fernández Enguita \& Terrén, 2008). Pero, además de los conceptos de trabajo en red, cooperación y gestión educativa abordados aquí, es necesario vislumbrar en esta línea de investigación nuevos objetos de estudio sobre este tipo de coordinación entre escuela y familias en entornos y comunidades virtuales (Sotomayor, 2014), respecto a la gestión del conocimiento (Rodríguez-Gómez, 2015), así como en relación al liderazgo docente (Contreras, 2016) o al papel que pueden jugar los Ayuntamientos (Muñoz \& Tolosana, 2010) en la construcción de la educación desde la escuela y las familias y con ellas.

Para acabar, comentamos que las limitaciones más notables de la contribución están asociadas a las posibilidades de generalización de las experiencias. Por consiguiente, su extensión a otras realidades debiera hacerse desde las adaptaciones pertinentes según cada situación, contexto y agentes implicados. De todas formas, ello no exime el que puedan servir de orientación para diseñar y desarrollar otras acciones de trabajo en red y cooperación entre escuela y familias a fin de mejorar la educación.

\section{Referencias}

Ajuntament de Ripollet. (2016). Esport i Escola: Un nou model d'esport escolar. Ripollet: Patronat Municipal d'Esports.

Apple, M., \& Beane, J. A. (Coords.). (2005). Escuelas democráticas. Madrid: Morata. 
Bassedas, E. (2007). La colaboración entre profesionales y el trabajo en red. En J. Bonals \& M. Sánchez (Coords.), Manual de asesoramiento psicopedagógico (pp. 43-66). Barcelona: Graó.

Blázquez, A. (2013). La información y comunicación: claves para la gestión del conocimiento empresarial. Dimens Empres, 1(11), 23-32.

Bolívar, A. (2005). La ciudadanía a través de la educación. Seminario 2005 año europeo de la ciudadanía a través de la educación. Madrid: Ministerio de Educación y Ciencia.

Comisionado para el Polígono Sur. (2010). Plan Educativo de Zona para el Polígono Sur 2010. Sevilla: Delegación del Gobierno en Andalucía, Junta de Andalucía y Ayuntamiento de Sevilla.

Contreras, T. (2016). Liderazgo pedagógico, liderazgo docente y su papel en la mejora de la escuela: una aproximación teórica. Propósitos y Representaciones, 4(2), 231-284. doi: 10.20511/pyr2016.v4n2.123

Egido, I. (2015). Las relaciones entre familia y escuela. Una vision general. Participación Educativa, 4(7), 11-18.

Epstein, J. L. (1990). School and family connections: Theory, research and implications for integrating sociologies of education and family. Marriage \& Family Review, 1-2(15), 99-126. doi: 10.1300/J002v15n01_06

Esteban, A. (2015). Relación familia-escuela: estudio descriptivo derivado de una experiencia de investigación-acción en el aula (Tesis Doctoral). Valladolid: Universidad de Valladolid.

Fernández Enguita, M. (2012). ¿Qué hay de nuevo bajo el sol? De las organizaciones y los grupos a las redes. Cuadernos de pedagogía, 385 , 12-19.

Fernández Enguita, M., \& Terrén, E. (Coords.). (2008). Repensando la organización escolar. Crisis de legitimidad y nuevos desarrollos. Madrid: AKAL.

Fernández, J. (1997). La participación en la comunidad educativa. Escuela Española, 3315(405), 17-19.

Gairín, J. (2004). Redes institucionales y de aprendizaje en la educación no formal. En Libro de Actas de las IX Jornadas Andaluzas de Organización 
y Dirección de Instituciones Educativas (pp. 23-46). Granada: Grupo Editorial Universitario.

Gairín, J. (2004a). Familia y escuela, un binomio inseparable. En $X X$ Congreso Interamericano de Educación Católica: Al reencuentro con la familia (pp. 89-147). Santiago de Chile: CIEC.

Gairín, J. (1998). L'escola. Ir Congreso de la Ciudad. Sant Cugat del Vallès: Fundació Sant Cugat del Vallès.

Gairín, J., Castro, D., Díaz, A., \& Muñoz, J. L. (2012). Codi de conducta per a competicions esportives escolars. Bellaterra: EDO-UAB y Consejo Superior de Deportes.

Gairín, J., \& San Fabián, J. L. (2005). La participación social en educación. En B. Jiménez (Coord.), Formación profesional (pp. 157-188). Barcelona: Praxis.

García, J., \& García, Á. (2001). Teoría de la Educación II. Procesos primarios de formación del pensamiento y la acción. Salamanca: Ediciones Universidad de Salamanca.

Hargreaves, A., (2003). Enseñar en la sociedad del conocimiento. Barcelona: Octaedro.

Johnson, D., Johnson, R., \& Holubec, E. (1999). El aprendizaje cooperativo en el aula. Argentina: Paidós.

Joyce, B., \& Weil, M. (2002). Modelos de enseñanza. Barcelona: Gedisa.

Koper, R. (Ed.). (2009). Learning network services for profesional development. Berlín: Heidelberg Springer.

Lozano, J., Castillo, I. S., Cerezo, Mª C., Angosto, R. \& Alcaraz, S. (2014). La participación de las familias en contextos educativos multiculturales: hacia una escuela intercultural e inclusiva. En R. Nortes \& J. I. Alonso (Eds.), Investigación educativa en educación primaria (pp. 218-229). Murcia: Edit.um.

Llevot, N. \& Bernad, O. (2015). La participación de las familias en la escuela: factores clave. Revista de la Asociación de Sociología de la Educación, $8(1), 57-70$.

Martín, Q. (2000). Bancos de talento. Participación de la comunidad en los centros docentes. Madrid: Sanz y Torres. 
Martín, M., \& Gairín, J. (Coords.). (2006). La participación en educación: los consejos escolares, una vía de participación. Santiago de Chile: Fundación Creando Futuro.

Martínez, J. B. (Coord.). (1998). Evaluar la participación en los centros educativos. Madrid: Escuela Española.

Mayordomo, A. (Coord.). (1999). Estudios sobre participación social en la enseñanza. Castelló: Diputació de Castelló.

McDonald, B. (1995). La evaluación como servicio público: perspectivas de futuro. En M $\mathrm{M}^{\mathrm{a}}$. J. Sáez (Ed.), Conceptualizando la evaluación en España (pp. 15-23). Alcalá de Henares: Servicio de Publicaciones de la Universidad de Alcalá.

MECD. (2015). Las relaciones entre familia y escuela. Experiencias y buenas prácticas. Madrid: Ministerio de Educación, Cultura y Deporte.

Mir, C. (Coord.). (1998). Cooperar en la escuela. La responsabilidad de educar para la democracia. Barcelona: Graó.

Morin, E. (2001). Tenir el cap clar. Barcelona: La campana.

Muñoz, J. L. (2014). La colaboración de la ciudadanía en la mejora de los centros educativos. International Journal of Educational Research and Innovation, 2(1), 98-106.

Muñoz, J. L. (2012). Ayuntamientos y desarrollo educativo. Madrid: Editorial Popular.

Muñoz, J. L. (2009). La participación de los municipios en la educación. Madrid: Editorial Popular.

Muñoz, J. L. \& Tolosana, C. (2010). El liderazgo de los municipios en la educación. En J. Gairín \& D. Castro (Eds.), Desarrollo educativo al servicio del desarrollo social (pp. 15-30). Santiago de Chile: FIDECAP - AECID.

Muñoz, J. L., Rodríguez, D., \& Barrera, A. (2013). Herramientas para la mejora de las organizaciones educativas y su relación con el entorno. Perspectiva Educacional, 25(1), 97-123.

Nagles, N. (2007). La gestión del conocimiento como fuente de innovación. Revista EAN, 61, 77-88. 
Ordóñez, R. (2015). La familia y la escuela ante los nuevos estilos de vida familiar. Kikiriki, 38, 14-15.

Páez, R. M. (2015). Familia y escuela: una alianza necesaria en la gestión escolar. Revista Iberoamericana de Educación, 67, 159-180.

Pujolàs, P. (2004). Aprender juntos alumnos diferentes. Los equipos de aprendizaje cooperativo en el aula. Barcelona: EUMO-Octaedro.

Revilla, E. \& Pérez, P. (1988). De la organización que aprende hacia la gestión del conocimiento. En VIII Congreso Nacional de ACEDE. Las Palmas de Gran Canaria.

Rodríguez-Gómez, D. (2015). Gestión del conocimiento y mejora de las organizaciones educativas. Madrid: La Muralla.

Rudduck, J. \& Flutter, J. (2007). Cómo mejorar tu centro escolar dando la voz al alumnado. Madrid: Morata.

Santos Guerra, M. Á. (2009). Escuelas para la democracia. Cultura, organización y dirección de instituciones educativas. Barcelona: Wolters Kluwer.

Senge, P. (1990). The fifth discipline, the art and practice of the learning organisation. New York: Doubleday.

Slavin, R. (1999). Aprendizaje cooperativo. Teoría, investigación y práctica. Argentina: Aique.

Soler, A. (2006). La escuela, núcleo de la red educativa territorial. Aula de Innovación Educativa, 152, 29-31.

Sotomayor, G. (2014). Comunidades virtuales de aprendizaje colaborativo para la educación superior. Propósitos y Representaciones, 2(2), 231303. doi: 10.20511/pyr2014.v2n2.64

Suárez, C. (2011). Asincronía, textualidad y cooperación en la formación virtual. Revista Española de Pedagogía, 249(69), 339-355.

Suárez, C. (2010). El aprendizaje cooperativo como condición social de aprendizaje. Barcelona: UOC.

Suárez, C. (2007). El potencial educativo de la interacción cooperativa. Investigación Educativa, 11(20), 61-78. 
Suárez, C., \& Gros, B. (2013). Aprender en red: de la interacción a la colaboración. Barcelona: UOC.

Ubieto, J. R. (2009). El trabajo en red: usos posibles en educación, salud mental y servicios sociales. Barcelona: Gedisa.

Vicent, C. (2014). Padres y maestros hacia el diálogo. Una perspectiva inglesa. Profesorado. Revista de Currículum y Formación del Profesorado, 2(18), 35-50.

Vigo, B., Dieste, B., \& Julve, C. (2016). Voces sobre participación de las familias en la escuela y éxito escolar. Revista de la Asociación de Sociología de la Educación, 3(9), 320-333.

Vigo, B., \& Julve, C. (2016). La participación de las familias en la escuela. Revista Electrónica Interuniversitaria de Formación del Profesorado, 53(19,1).

Vigotsky, L. (2000). El desarrollo de los procesos psicológicos superiores. Barcelona: Crítica. 